OPEN ACCESS

Edited by:

Chia-Ying Lee,

Academia Sinica, Taiwan

Reviewed by:

Gang Peng,

The Chinese University of Hong Kong,

Hong Kong

Jing Zhao,

Capital Normal University, China

*Correspondence:

Linjun Zhang

zhanglinjun75@gmail.com

Specialty section:

This article was submitted to

Language Sciences,

a section of the journal

Frontiers in Psychology

Received: 12 March 2017 Accepted: 13 June 2017

Published: 28 June 2017

Citation:

Zhou H, Li Y, Liang M, Guan CQ, Zhang L, Shu H and Zhang Y (2017)

Mandarin-Speaking Children's Speech Recognition: Developmental

Changes in the Influences

of Semantic Context and $F_{0}$

Contours. Front. Psychol. 8:1090.

doi: 10.3389/fpsyg.2017.01090

\section{Mandarin-Speaking Children's Speech Recognition: Developmental Changes in the Influences of Semantic Context and $\boldsymbol{F}_{0}$ Contours}

\author{
Hong Zhou', Yu Li' ${ }^{2}$, Meng Liang ${ }^{3}$, Connie Qun Guan ${ }^{4}$, Linjun Zhang ${ }^{3 *}$, Hua Shu ${ }^{5}$ and \\ Yang Zhang ${ }^{6}$ \\ 1 International Cultural Exchange School, Shanghai University of Finance and Economics, Shanghai, China, ${ }^{2}$ Department of \\ Cognitive Science and ARC Centre of Excellence in Cognition and Its Disorders, Macquarie University, Sydney, NSW, \\ Australia, ${ }^{3}$ College of Allied Health Sciences, Beijing Language and Culture University, Beijing, China, ${ }^{4}$ School of Foreign \\ Studies, University of Science and Technology Beijing, Beijing, China, ${ }^{5}$ National Key Laboratory of Cognitive Neuroscience \\ and Learning, Beijing Normal University, Beijing, China, ${ }^{6}$ Department of Speech-Language-Hearing Sciences and Center for \\ Neurobehavioral Development, University of Minnesota, Minneapolis, MN, United States
}

The goal of this developmental speech perception study was to assess whether and how age group modulated the influences of high-level semantic context and lowlevel fundamental frequency $\left(F_{0}\right)$ contours on the recognition of Mandarin speech by elementary and middle-school-aged children in quiet and interference backgrounds. The results revealed different patterns for semantic and $F_{0}$ information. One the one hand, age group modulated significantly the use of $F_{0}$ contours, indicating that elementary school children relied more on natural $F_{0}$ contours than middle school children during Mandarin speech recognition. On the other hand, there was no significant modulation effect of age group on semantic context, indicating that children of both age groups used semantic context to assist speech recognition to a similar extent. Furthermore, the significant modulation effect of age group on the interaction between $F_{0}$ contours and semantic context revealed that younger children could not make better use of semantic context in recognizing speech with flat $F_{0}$ contours compared with natural $F_{0}$ contours, while older children could benefit from semantic context even when natural $F_{0}$ contours were altered, thus confirming the important role of $F_{0}$ contours in Mandarin speech recognition by elementary school children. The developmental changes in the effects of high-level semantic and low-level $F_{0}$ information on speech recognition might reflect the differences in auditory and cognitive resources associated with processing of the two types of information in speech perception.

Keywords: semantic context, fundamental frequency contours, speech recognition, interfering speech, children

\section{INTRODUCTION}

Speech recognition involves smooth and rapid integration of perceptual and cognitive systems, during which lower- and higher-level information sources in the spoken language are analyzed and synthesized (Moore et al., 2008). In adverse listening conditions, acoustic-phonetic cues are often insufficient for the identification of particular words because of speech signal quality (e.g., distortion to speech sounds caused by poor articulation) or listening background (e.g., the 
presence of noise or interfering speech). In such circumstances, various supportive mechanisms, especially the semantic context from the preceding words in an utterance or discourse can help the listener perceive, recognize, and understand subsequent words. Previous studies have shown that these factors including quality of speech signal, listening background, and semantic context interact with each other in jointly affecting speech recognition outcome (Pichora-Fuller et al., 1995; Sheldon et al., 2008; Benichov et al., 2012). For example, Laures and Bunton (2003) found that acoustically degraded sentences with flattened fundamental frequency (the lowest frequency of a periodic complex sound and is the acoustic correlate of pitch for speech and music, hereafter referred to as $F_{0}$ ) contours were as intelligible as original sentences with natural $F_{0}$ contours when both sentence types were presented in quiet. However, the flattened $F_{0}$ contours yielded decreased speech intelligibility in white noise and multi-talker babble backgrounds, indicating the significant interaction between the quality of speech signal and listening background during speech recognition. Dubno et al. (2000) showed that both older and young adults derived greater contextual benefit for recognizing the sentence-final word in the presence of masking broadband noise when compared with quiet, indicating the significant interaction between listening condition and semantic context for speech intelligibility.

The task of listening to speech under suboptimal circumstances is challenging for listeners of all ages, but the detrimental effects of degraded speech and auditory masking appear to be particularly large during childhood because children are not born with fully mature auditory processing capabilities but instead demonstrate a developmental time course of improvement in processing complex auditory situations (Eisenberg et al., 2000; Hall et al., 2002; Blandy and Lutman, 2005; Stuart, 2005; Wilson et al., 2010). Children's difficulty in perceiving speech in noise and against interfering speech has been investigated in a number of previous studies, which have consistently shown that children need more favorable conditions than adults to achieve comparable speech-in-noise scores (Papso and Blood, 1989; Fallon et al., 2000; Stuart et al., 2006). For example, Fallon et al. (2000) examined sentence recognition by young adults and children of three age groups $(5,9$, and 11 years) in the presence of an eight-talker babble and found that children of all the three groups required a more favorable signalto-noise ratio (SNR) to perform as well as the young adults. This child-adult difference was largest for the youngest children, reflecting the developmental progress in the ability to recognize speech in multi-talker babbles. Stuart (2005) suggested a similar disadvantage for children in speech recognition in noise when comparing the performances of 6- to 15-year-old children and young adults on the NU-CHIPS (the Northwestern University Children's Perception of Speech test) in quiet, steady-state and interrupted background noise. The younger children exhibited greater difficulty for listening in both kinds of background noise than the older children and adult-like performance was only exhibited in the children 11 years of age and older.

Previous work suggests that younger children are able to use semantic context to assist speech recognition in quiet as early as 2 -years-old involving predictive coding to recognize the sentence-final nouns (Fernald, 2001). The ability to use semantic context in adverse listening backgrounds continues to improve into adolescence with significant interactions among the factors of listening background, semantic context and age. For example, investigation of speech intelligibility of high- and lowpredictability sentences in children at 9-17 years of age showed that contextual cues assisted children of all ages in identifying words masked by babbles with older children performing better than younger children in the high context conditions (Elliot, 1979). Similar studies including children aged $5-11$ years (Fallon et al., 2000) or 5-10 years (Stelmachowicz et al., 2000) also supported age-related improvement in contextual benefit for recognizing speech in adverse listening backgrounds.

In addition to the line of work on the effects of adverse listening backgrounds, there has been another line of investigation on the effect of degraded speech signal on speech recognition by children. Smiljanic and Sladen (2013) found that clear speech with more pauses, longer sentence/vowel durations and greater vowel spaces was more intelligible than conversational speech for children aged 6-13 years. In addition, children have the ability to use semantic context to decode degraded signals in quiet. For example, in a quiet listening condition, Cole and Perfetti (1980) found that 4-years-old were better at detecting mispronounced words in high-predictability sentences than in low-predictability sentences. Craig et al. (1993) found that children 8-10 years of age required shorter portions of the target words (i.e., less acoustic-phonetic information) for recognition in high-predictability than in low-predictability sentences. By contrast, children aged 5-7 years required as much acoustic-phonetic information with highpredictability as with low-predictability contexts to identify target words.

To our knowledge, previous studies have not combined the two lines of work to investigate whether children can use sentence context to aid recognition of degraded speech in adverse listening backgrounds. There is also a lack of studies in which the primary target language is not English to test the generalizability of previous findings on other languages with typologically distinct acoustic and prosodic properties. In a tonal language like Mandarin Chinese, $F_{0}$ is primarily used to distinguish lexical meanings (Wang, 1973). This differs greatly from non-tonal languages (e.g., English and French) in which $F_{0}$ information is mainly used for pragmatic purposes such as sentence modality (interrogative vs. declarative), emphasis and emotion (Cutler et al., 1997). Although lexical tones have a phonemic role in Mandarin, alteration of the original $F_{0}$ contours has little effect on the intelligibility of sentences in quiet. In contrast, adverse listening conditions (e.g., interfering speech) substantially reduce the intelligibility of Mandarin sentences with altered $F_{0}$ contours compared with sentences with natural $F_{0}$ patterns (Wang et al., 2013; Chen et al., 2014). Developmental research reveals that Mandarin-speaking children show sensitivity to $F_{0}$ information several days after birth (Wermke et al., 2016) and acquire the $F_{0}$ patterns of lexical tones in perception and production at an early age (Hua and Dodd, 2000). Considering the interaction of sentential semantic context, $F_{0}$ contours and listening condition on Mandarin speech recognition by young 
adults (Wang et al., 2013), it remains unclear how these factors may separately or jointly affect a child's performance.

The purpose of the present study aimed to investigate the possible different effects of high-level semantic and low-level $F_{0}$ information on the recognition of Mandarin speech by elementary and middle school children in quiet and single-talker interference conditions. On the basis of previous studies that have suggested a developmental/maturational course for children's ability to recognize speech in adverse listening conditions (Elliot, 1979; Stelmachowicz et al., 2000; Stuart, 2005), we predicted that the middle school-aged children would perform better than the elementary school children in the presence of interfering speech, while the two groups would perform equally well in quiet. Furthermore, the middle school-aged children would benefit more from sentence context than the elementary school children in the interfering background but not in quiet. More importantly, we expected a significant interaction between age group and $F_{0}$ contours. Specifically, the middle school children would perform better than the elementary school children when $F_{0}$ contours were flattened, while both groups would perform similarly on recognizing speech with natural $F_{0}$ contours, assuming that younger children might rely more on natural $F_{0}$ patterns than older children in speech recognition. The effect of age group on $F_{0}$ contours might be further modulated by listening background and semantic context, e.g., the middle school children could benefit more from semantic context than the elementary school children when $F_{0}$ patterns changed from natural contours to flat contours.

\section{MATERIALS AND METHODS}

\section{Subjects}

Forty-eight elementary school children (23 boys, Grade 4, age range 9.6-10.1 years) and forty-six middle school children (25 boys, Grade 8, age range 13.5-14.2 years) from local schools in Beijing were recruited. All children were native speakers of Mandarin Chinese and none of the children had a history of any neurological or psychiatric disorders. Audiometric evaluation was carried out using a GSI 61 clinical audiometer (GrasonStadler, Inc., Madison, WI, United States) with a standard Hughson-Westlake approach and each child had pure-tone thresholds no greater than $20 \mathrm{~dB} \mathrm{HL}$ at octave intervals from 250 to $8000 \mathrm{~Hz}$ bilaterally. Written informed consent was obtained from the children and their legal guardians. The study was approved by the Institutional Review Boards (IRBs) at Beijing Language and Culture University and Beijing Normal University, and was conducted in compliance with the Declaration of Helsinki.

This study focused on the two age groups because children 11 years of age and older exhibited adult-like performance on speech recognition in noise (Stuart, 2005). A mixed-design was adopted with semantic context (normal sentence vs. word list sentence) and $F_{0}$ contours (normal vs. flat) as within-subject factors, while listening background (quiet vs. interference) and age group (elementary school vs. middle school) as betweensubject factors. The participants (boys/girls) randomly divided in the 4 between-subject conditions were distributed as follows: elementary school/quiet, number of subjects $(n)=24$ including 11 boys and 13 girls; elementary school/interference background, $n=24$ including 12 boys and 12 girls; middle school/quiet, $n=23$ including 13 boys and 10 girls; middle school/interference background, $n=23$ including 12 boys and 11 girls.

\section{Stimuli}

Four types of sentences were used as targets. Specifically, the target sentences included normal/word list sentences with natural/flat $F_{0}$ contours, respectively, manipulating semantic context and $F_{0}$ contours. Such manipulations have been adopted in our previous studies (Wang et al., 2013; Zhang et al., 2016; Jiang et al., 2017). The normal sentences were 28 declarative Chinese sentences with each sentence comprised of 3 to 6 words (2-4 content words plus $0-2$ functional words) that were familiar to both the elementary and middle school children. Words from the entire pool of the normal sentences were pseudo-randomly selected to form the word list sentences matched in number of content and functional words with the normal sentences. Word list sentences were syntactically correct but semantically meaningless at the whole sentence level. That is, recognition of both word list and normal sentence recruits syntactic and semantic processing, but only normal sentence provides the meaningful semantic context of "who does what to whom" beyond random lexical semantics. A male native Chinese speaker read the normal and word list sentences. Monotonous sentences with flat $F_{0}$ contours were created by flattening the natural $F_{0}$ contours at each sentence's mean $F_{0}$ (Figure 1).

Masker stimuli were consonant-misplaced sentences, which were constructed based on the normal sentences. Specifically, the initial consonant of every syllable in each normal sentence was replaced with another consonant as long as the phonotactic rules of Mandarin were not violated. Consonant-misplaced sentences were unintelligible at both word and sentence levels, thus having minimal effect of informational masking (Wang et al., 2013; Xu et al., 2013). A female native Mandarin speaker read the masker sentences, which was to enable separation of the target message from the interfering speech. Without this indexical information, participants would have to be trained on the identity of the target speaker. The listeners were instructed to listen to the male speaker for the content rather than the identity of the target speaker throughout (Scott et al., 2004). The target and masker sentences were first edited to be at the sound pressure levels of 75 and $70 \mathrm{~dB}$, respectively, and then combined to form the speech against interference stimuli with SNR level set at $+5 \mathrm{~dB}$.

\section{Procedures}

We followed similar experimental procedures in our previous studies (Wang et al., 2013; Zhang et al., 2016), except that listeners were instructed to verbally repeat rather than write down the whole target normal/word list sentences in order to reduce cognitive burden on the children. Their responses were recorded and scored by the first author of this paper and checked by an independent auditor blind to the experiment. A strict score standard was adopted. Specifically, only words with consonants, vowels and lexical tones all correctly identified were considered 

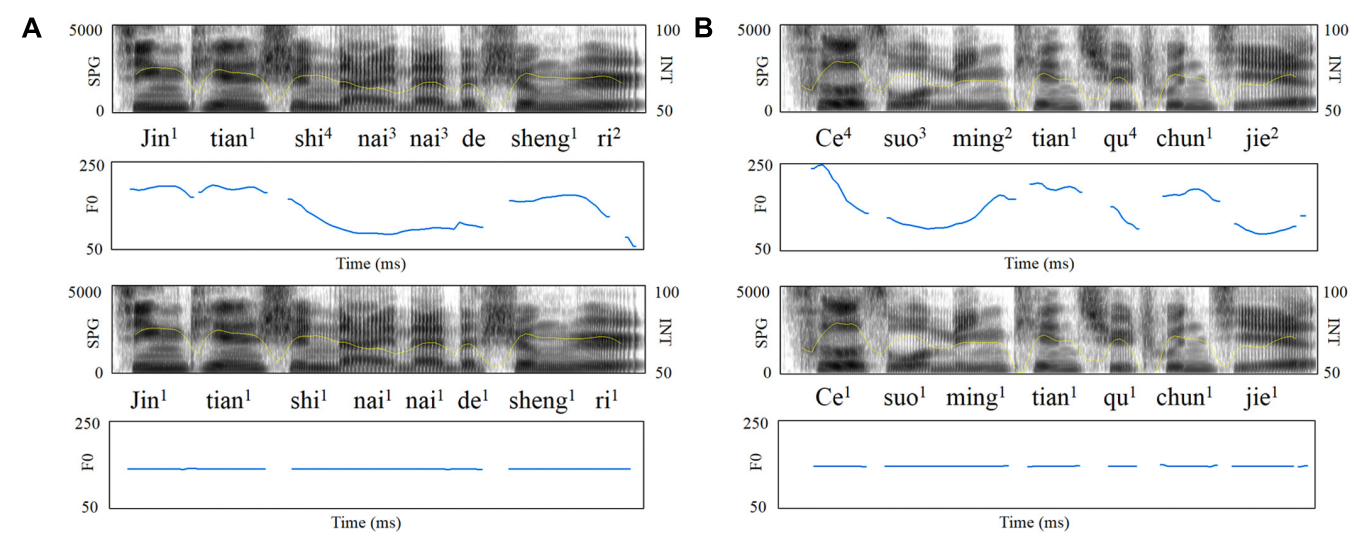

FIGURE 1 | Acoustic features of sample speech stimuli. Broadband spectrograms (SPG: 0-5 kHz), intensity envelopes (INT: 50-100 dB), and fundamental frequency contours (FO: 0-300 Hz) are displayed for (A) normal sentence and its pitch-flattened counterpart; (B) word list sentence and its pitch-flattened counterpart.

correct answers. During the experiment, the listeners were seated in a sufficiently silent room (not higher than $15 \mathrm{~dB}$ for ambient noise level). A set of Edifier R18 loudspeakers were used to present the stimuli with the sound level set at $65 \mathrm{~dB}$ SPL calibrated at the listeners' head. Because $F_{0}$ contours and semantic context were within-subject factors, each listener was presented with a total of 56 trials -14 normal sentences and 14 word list sentences with natural or flat $F_{0}$ contours. Each child listened to half of the total normal sentences/wordlist sentences (14/28) with natural $F_{0}$ contours and the other half with flat $F_{0}$ contours, i.e., each child did not listen to the same normal sentence/wordlist sentence with natural or flat $F_{0}$ contours. $F_{0}$ patterns were counterbalanced across subjects. The experiment adopted self-paced paradigm and the children were encouraged to guess which words they heard. Each normal/word list sentence was used only once with each subject. Before the actual experiment, all children participated in a brief practice session representing samples of all conditions.

\section{RESULTS}

A keyword-correct count was used to calculate the speech recognition sore, i.e., percentage of content words correctly

TABLE 1 | Mean accuracy ( \pm standard deviation) of each condition for both groups.

\begin{tabular}{|c|c|c|c|c|}
\hline & \multicolumn{2}{|c|}{ Natural $F_{0}$} & \multicolumn{2}{|c|}{ Flat $F_{0}$} \\
\hline & $\begin{array}{l}\text { Normal } \\
\text { sentence }\end{array}$ & $\begin{array}{l}\text { Word list } \\
\text { sentence }\end{array}$ & $\begin{array}{l}\text { Normal } \\
\text { sentence }\end{array}$ & $\begin{array}{l}\text { Word list } \\
\text { sentence }\end{array}$ \\
\hline & \multicolumn{4}{|c|}{ Elementary school children } \\
\hline Quiet & $0.94( \pm 0.05)$ & $0.84( \pm 0.08)$ & $0.82( \pm 0.12)$ & $0.66( \pm 0.13)$ \\
\hline \multirow[t]{2}{*}{$+5 \mathrm{~dB}$ SNR } & $0.83( \pm 0.12)$ & $0.63( \pm 0.12)$ & $0.54( \pm 0.14)$ & $0.39( \pm 0.16)$ \\
\hline & \multicolumn{4}{|c|}{ Middle school children } \\
\hline Quiet & $0.97( \pm 0.05)$ & $0.90( \pm 0.05)$ & $0.92( \pm 0.04)$ & $0.77( \pm 0.10)$ \\
\hline$+5 \mathrm{~dB}$ SNR & $0.89( \pm 0.08)$ & $0.72( \pm 0.13)$ & $0.75( \pm 0.14)$ & $0.54( \pm 0.13)$ \\
\hline
\end{tabular}

identified (Scott et al., 2004; Wang et al., 2013) (Table 1). In order to test the main and interaction effects, a repeated measures ANOVA was conducted with listening condition and age group as between-subject factors, and sentence context and $F_{0}$ contours as within-subject factors. Results revealed significant main effects of all the four factors [semantic context: $F(1,90)=275.877, p<0.001, \eta_{\mathrm{p}}^{2}=0.754$; listening background: $F(1,90)=112.168, p<0.001, \eta_{\mathrm{p}}^{2}=0.555 ; F_{0}$ contours: $F(1,90)=384.19, p<0.001, \eta_{\mathrm{p}}^{2}=0.81$; age group, $\left.F(1,90)=32.236, p<0.001, \eta_{\mathrm{p}}^{2}=0.264\right]$, and four significant two-way interaction effects as well [interaction between semantic context and listening condition: $F(1,90)=12.53, p<0.001$, $\eta_{\mathrm{p}}^{2}=0.122$; interaction between $F_{0}$ contours and age group: $F(1,90)=22.464, p<0.001, \eta_{\mathrm{p}}^{2}=0.2$; interaction between listening background and $F_{0}$ contours: $F(1,90)=32.48$, $p<0.001, \eta_{p}^{2}=0.265$; and interaction between $F_{0}$ contours and semantic context: $\left.F(1,90)=4.866, p=0.03, \eta_{\mathrm{p}}^{2}=0.051\right]$.

There were also two significant 3-way interaction effects [interaction between age group, $F_{0}$ contours and semantic context: $F(1,90)=5.271, p=0.024, \eta_{\mathrm{p}}^{2}=0.055$, and interaction between $F_{0}$ contours, semantic context and listening background: $\left.F(1,90)=7.292, p=0.008, \eta_{\mathrm{p}}^{2}=0.075\right]$. The four-way interaction effect was not significant $\left[F(1,90)=1.155, p=0.285, \eta_{p}^{2}=0.013\right]$. Considering the primary purpose of the present study was to investigate the developmental difference between elementary and middle school children in the use of semantic context and $F_{0}$ contours during Mandarin speech recognition, the following analyses focused on the significant 3-way interaction between age group, $F_{0}$ contours and semantic context. Specifically, this interaction effect was decomposed step by step (Maxwell and Delaney, 2004). First, separate two-way ANOVAs were carried out to examine the simple interaction effects. Results showed that significant interaction between $F_{0}$ contours and semantic context was observed in the middle school children $[F(1,45)=7.837$, $\left.p=0.008, \eta_{\mathrm{p}}^{2}=0.151\right]$, but absent in the elementary school children $\left[F(1,47)=0.006, p=0.941, \eta_{\mathrm{p}}^{2}<0.001\right]$ (Figure 2). Second, follow-up analyses were carried out on the simple 
interaction effects to examine the simple main effects. Results showed that for both the elementary and middle school children, normal/word list sentence with natural $F_{0}$ contours were better recognized than their counterparts with flat $F_{0}$ contours; normal sentence with natural/flat $F_{0}$ contours were better recognized than their word list counterparts [elementary school children: $F(1,47)>63.096, p<0.001, \eta_{\mathrm{p}}^{2} \geq 0.573$; middle school children: $\left.F(1,45)>32.801, p<0.001, \eta_{\mathrm{p}}^{2} \geq 0.422\right]$.

\section{DISCUSSION}

The present developmental study explored Mandarin speech recognition by children beyond the age of 9 . Two distinct patterns emerged in assessing whether and how age group modulate the influences of low-level $F_{0}$ contours and high-level semantic context on Mandarin speech recognition in quiet and interference backgrounds. Specifically, there was a significant modulation effect of age group on $F_{0}$ contours, indicating that the elementary school children relied more on natural $F_{0}$ contours than the middle school children during Mandarin speech recognition. By contrast, there was no significant modulation effect of age group on semantic context, indicating that children of both age groups used semantic context to assist speech recognition to a similar extent. Furthermore, the significant modulation effect of age group on the interaction between $F_{0}$ contours and semantic context revealed that younger children could not make better use of semantic context in recognizing speech with flat $F_{0}$ contours compared with natural $F_{0}$ contours, while older children benefited more from semantic context when natural $F_{0}$ contours were altered, thus confirming the important role of $F_{0}$ contours in Mandarin speech recognition by the elementary school children.

Children's ability to recognize speech in suboptimal listening backgrounds improves with age (Elliot, 1979; Stuart, 2005), and their ability to use semantic context to assist speech recognition also shows a developmental change from childhood to adolescence (Fallon et al., 2000; Stelmachowicz et al., 2000). For the present study that focused on elementary and middle school-aged children, our results showed that both groups of children performed better on speech recognition in quiet than in the interference background and that both groups benefited from semantic context to a similar degree regardless of listening backgrounds. These results indicate that Mandarin-speaking children 9.6-10.1 of age (Grade 4) have developed the ability to recognize speech against an interfering talker as well as the ability to use semantic context to assist speech recognition, both of which are comparable with those of children 13.5-14.2 of age (Grade 8). Because only two age groups of children were included in this study, it is unclear at what age children start to develop such abilities comparable with the middle school-aged children.

Although recognition of degraded speech by children has been investigated in several previous studies (e.g., Smiljanic and Sladen, 2013), how alterations in natural $F_{0}$ contours affect speech intelligibility for children has rarely been investigated. Natural $F_{0}$ contours play an important role in speech recognition because dynamic changes in natural $F_{0}$ contours help listeners
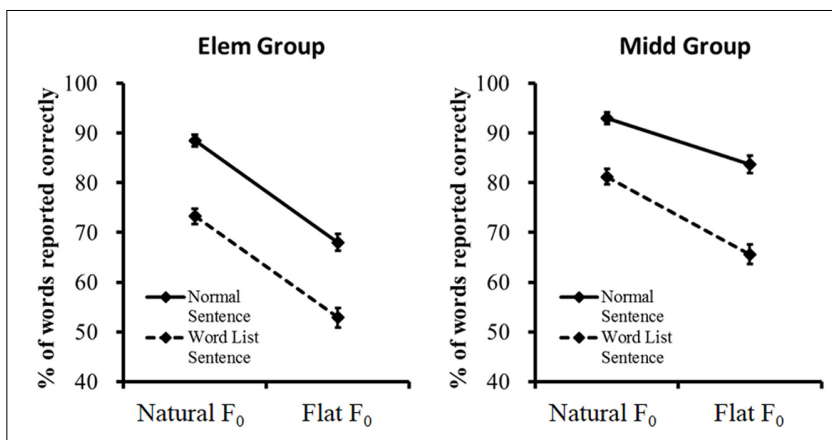

FIGURE 2 | Word-report scores of the simple interaction effects conducted on the significant 3-way interaction between age group, $F_{0}$ contours and semantic context. Error bars represent standard deviation across subjects.

recognize voice/unvoiced phonetic segments, separate words in continuous speech and focus attention on the content words. Infants are sensitive to $F_{0}$ patterns of speech. For example, newborns perceive emotion conveyed via $F_{0}$ contours in maternal speech (Fernald and Simon, 1984) and distinguish between languages based on prosodic information (Nazzi et al., 1998; Ramus et al., 2000). Even newborns' cry melody is influenced by $F_{0}$ patterns of their native languages (Mampe et al., 2009). Our finding that recognition of speech with flat $F_{0}$ contours degraded to a greater extent in the elementary school children than in the middle school children indicate that younger children rely more on natural $F_{0}$ contours to assist speech recognition. One plausible explanation is that flattening natural $F_{0}$ contours alters the acoustic-phonetic features of some speech segments and contrasts between words and makes it more difficult for younger children to parse continuous speech into meaningful units, while older children are likely to have developed highly effective and efficient strategies for compensating for the detrimental effects by focusing their attention on the cues that are less vulnerable to $F_{0}$ distortion. An alternative explanation is that the monotonous speech with flat $F_{0}$ contours are not attractive enough to the younger listeners, thus resulting in additional costs related to attentional abatement when compared with the older children.

Previous work on young adults shows that $F_{0}$ contours affect speech recognition interactively with listening background and semantic context. In particular, flattening or inverting $F_{0}$ contours lowers speech intelligibility to a larger degree in adverse listening backgrounds than in quiet no matter whether the target language is a non-tonal or tonal language; the detrimental effect, however, is more serious for a tonal language (Binns and Culling, 2007; Miller et al., 2010; Patel et al., 2010). In addition, alteration in $F_{0}$ contours deteriorates speech intelligibility of low-predictability/word list sentences more than high-predictability/normal sentences (Wang et al., 2013; Chen et al., 2014). In the present study, we found a significant modulation effect of age on the interaction between $F_{0}$ contours and semantic context, but the interaction between $F_{0}$ contours and listening background was not modulated by age. Specifically, for the elementary school children, semantic context did not provide extra benefit when $F_{0}$ contours were flattened 
compared with speech with natural $F_{0}$ contours. However, for the middle school children, semantic context did provide extra benefit when $F_{0}$ contours were flattened. These results seem to indicate that the middle school-aged children could use semantic context to compensate for the degraded speech signal caused by flat $F_{0}$ contours, while this ability is not well developed in the elementary school children. By contrast, both age groups of children benefited to a similar extent from semantic context when the listening background changes from quiet to interference condition, which indicates that the elementary and middle school children are capable of using semantic context to resist interfering speech. Speech recognition by young and older adults in quiet and single-talker interference backgrounds has been explored in our previous studies (Wang et al., 2013 for young adults, and Jiang et al., 2017 for older listeners). Although it is unjustifiable to make a direct statistical comparison across the results of different age groups because different word materials and elicitation approaches were adopted, it is meaningful to compare the different patterns of interaction effects between semantic context and $F_{0}$ contours/listening background across the four age groups. For the middle school children and young adults, interaction effects between semantic context and $F_{0}$ contours/listening background are both significant, indicating that the two groups could use semantic context to compensate for the distorted speech signal caused by flat $F_{0}$ contours and interfering speech. Interestingly, for the elementary school children, only the interaction effect between semantic context and listening background is significant; while for the older listeners, only interaction effect between semantic context and $F_{0}$ contours is significant. The modulation effects of age on the interactions between semantic context and $F_{0}$ contours/listening background are likely to be related to the maturational/decaying process of speech recognition with degraded signal. Our data indicate that the different age groups may have different receptive skills in processing distortions caused by competing speech and flattened $F_{0}$ contours. Competing speech results in acoustic and informational masking to the recognition task, while altering $F_{0}$ contours distorts the target speech signal. That is, competing speech and flattened $F_{0}$ contours introduces external and internal distortions, respectively, which may need to recruit different cognitive and neural processes during speech perception depending on the listeners' age. Specifically, it has been suggested that the downstream brain areas (e.g., bilateral inferior frontal regions) are involved in the processing of external distortions, whereas the upstream areas (e.g., left temporal regions) are responsible for the processing of internal distortions (Adank et al., 2012). The age-related differences in the use of semantic context to assist the recognition of different kind of distorted speech might be associated with the

\section{REFERENCES}

Adank, P., Davis, M. H., and Hagoort, P. (2012). Neural dissociation in processing noise and accent in spoken language comprehension. Neuropsychologia 50, 77-84. doi: 10.1016/j.neuropsychologia.2011. 10.024 developmental/aging changes in cortical/subcortical networks that process the target speech signal in the presence of the two kinds of signal degradation, which calls for further investigation in future studies.

The masker stimuli used in the current study are consonantmisplaced sentences that are syntactically anomalous and semantically incomprehensible, and thus primarily pose energetic (as opposed to informational) masking on the targets. One concern might arise with the specific masking effects on the current findings. Various kinds of maskers (e.g., broadband noise, one- and multi-talker babble) have different acoustic/phonetic properties and thus differ greatly in masking targets and distracting listeners. Further studies using different kinds of interfering sounds are necessary to clarify how children's speech recognition is affected by characteristics of maskers.

\section{CONCLUSION}

Our current results provide supporting evidence that children use semantic context to assist speech recognition in quiet and adverse listening backgrounds. Although the abilities to recognize speech in adverse listening backgrounds and to use semantic context to assist speech recognition appear to have well developed in normal children aged 9 and beyond, younger children depend more on natural $F_{0}$ contours in speech recognition than older children. These developmental changes in the influences of semantic context and $F_{0}$ contours might reflect the differences in auditory and cognitive resources associated with processing of the two types of information in speech perception.

\section{AUTHOR CONTRIBUTIONS}

Research design: HZ, YL, CQG, LZ, and HS. Data collection: HZ, ML, and YL. Data analysis: HZ, YL, and ML. Manuscript preparation: LZ, YZ, and HS.

\section{ACKNOWLEDGMENTS}

This research was supported by the Open Research Fund of the State Key Laboratory of Cognitive Neuroscience and Learning (CNLYB1607) to LZ, by the National Key Basic Research Program of China (2014CB846103), Natural Science Foundation of China (81461130018, 31671126, 31611130107) and Beijing Municipal Science and Technology Commission (Z151100003915122) to HS, and in part from the National Institute of Child Health and Human Development (P01HD001994) to Haskins Laboratories.

Benichov, J., Cox, L. C., Tun, P. A., and Wingfield, A. (2012). Word recognition within a linguistic context: effects of age, hearing acuity, verbal ability, and cognitive function. Ear Hear. 33, 250-256. doi: 10.1097/AUD.0b013e31822f680f

Binns, C., and Culling, J. F. (2007). The role of fundamental frequency contours in the perception of speech against interfering speech. J. Acoust. Soc. Am. 122, 1765-1776. doi: 10.1121/1.2751394 
Blandy, S. E., and Lutman, M. E. (2005). Hearing threshold levels and speech recognition in noise in 7-year-olds. Int. J. Audiol. 44, 435-443. doi: 10.1080/ 14992020500189203

Chen, F., Wong, L. L., and Hu, Y. (2014). Effects of lexical tone contour on Mandarin sentence intelligibility. J. Speech Lang. Hear. Res. 57, 338-345. doi: 10.1044/1092-4388

Cole, R. A., and Perfetti, C. A. (1980). Listening for mispronunciations in a children's story: the use of context by children and adults. J. Verbal Learn. Verbal Behav. 19, 297-315. doi: 10.1016/S0022-5371(80)90239-X

Craig, C. H., Kim, B. W., Rhyner, P. M., and Chirillo, T. K. (1993). Effects of word predictability, child development, and aging on time-gated speech recognition performance. J. Speech Hear. Res. 36, 832-841. doi: 10.1044/jshr.3604.832

Cutler, A., Dahan, D., and van Donselaar, W. (1997). Prosody in the comprehension of spoken language: a literature review. Lang. Speech 40, 141-201. doi: 10.1177/002383099704000203

Dubno, J. R., Ahlstrom, J. B., and Horwitz, A. R. (2000). Use of context by young and aged adults with normal hearing. J. Acoust. Soc. Am. 107, 538-546. doi: $10.1121 / 1.428322$

Eisenberg, L. S., Shannon, R. V., Martinez, A. S., Wygonski, J., and Boothroyd, A. (2000). Speech recognition with reduced spectral cues as a function of age. J. Acoust. Soc. Am. 107, 2704-2710. doi: 10.1121/1.428656

Elliot, L. L. (1979). Performance of children aged 9 to 17 years on a test of speech intelligibility in noise using sentence material with controlled word predictability. J. Acoust. Soc. Am. 66, 651-653. doi: 10.1121/1. 383691

Fallon, M., Trehub, S. E., and Schneider, B. A. (2000). Children's perception of speech in multitalker babble. J. Acoust. Soc. Am. 108, 3023-3029. doi: 10.1121/ 1.1323233

Fernald, A. (2001). Making use of semantic context in early language understanding. Paper Presented at the Society for Research in Child Development, Minneapolis, MN.

Fernald, A., and Simon, T. (1984). Expanded intonation contours in mothers' speech to newborns. Dev. Psychol. 20, 104-113. doi: 10.1037/0012-1649.20.1. 104

Hall, J. W., Grose, J. H., Buss, E., and Dev, M. B. (2002). Spondee recognition in a two-talker masker and a speech-shaped noise masker in adults and children. Ear Hear. 23, 159-165. doi: 10.1097/00003446-200204000-00008

Hua, Z., and Dodd, B. (2000). The phonological acquisition of Putonghua (modern standard Chinese). J. Child Lang. 27, 3-42.

Jiang, W., Li, Y., Hua, S., Zhang, L., and Zhang, Y. (2017). Use of semantic context and F0 contours by older listeners during Mandarin speech recognition in quiet and single-talker interference conditions. J. Acoust. Soc. Am. 141, EL338-EL344. doi: $10.1121 / 1.4979565$

Laures, J. S., and Bunton, K. (2003). Perceptual effects of a flattened fundamental frequency at the sentence level under different listening conditions. J. Commun. Disord. 36, 449-464. doi: 10.1016/S0021-9924(03)00032-7

Mampe, B., Friederici, A. D., Christophe, A., and Wermke, K. (2009). Newborns' cry melody is shaped by their native language. Curr. Biol. 19, 1994-1997. doi: 10.1016/j.cub.2009.09.064

Maxwell, S. E., and Delaney, H. D. (2004). Designing Experiments and Analyzing Data: A Model Comparison Perspective, 2nd Edn. Mahwah, NJ: Erlbaum.

Miller, S. E., Schlauch, R. S., and Watson, P. J. (2010). The effects of fundamental frequency contour manipulations on speech intelligibility in background noise. J. Acoust. Soc. Am. 128, 435-443. doi: 10.1121/1.3397384

Moore, B. C. J., Tyler, L. K., and Marslen-Wilson, W. (2008). Introduction. The perception of speech: from sound to meaning. Philos. Trans. R. Soc. Lond. B Biol. Sci. 363, 917-921. doi: 10.1098/rstb.2007.2195

Nazzi, T., Floccia, C., and Bertoncini, J. (1998). Discrimination of pitch contours by neonates. Infant. Behav. Dev. 21, 779-784. doi: 10.1016/S0163-6383(98)90044-3

Papso, C. F., and Blood, I. M. (1989). Word recognition skills of children and adults in background noise. Ear Hear. 10, 235-236. doi: 10.1097/00003446198908000-00004
Patel, A. D., Xu, Y., and Wang, B. (2010). "The role of F0 variation in the intelligibility of Mandarin sentences," in Proceedings of Speech Prosody 2010, Chicago, IL.

Pichora-Fuller, M. K., Schneider, B. A., and Daneman, M. (1995). How young and old adults listen to and remember speech in noise. J. Acoust. Soc. Am. 97, 593-608. doi: 10.1121/1.412282

Ramus, F., Hauser, M. D., Miller, C., Morris, D., and Mehler, J. (2000). Language discrimination by human newborns and by cotton-top tamarin monkeys. Science 288, 349-351. doi: 10.1126/science.288.5464.349

Scott, S. K., Rosen, S., Wickham, L., and Wise, R. J. (2004). A positron emission tomography study of the neural basis of informational and energetic masking effects in speech perception. J. Acoust. Soc. Am. 115, 813-821. doi: 10.1121/1. 1639336

Sheldon, S., Pichora-Fuller, M. K., and Schneider, B. A. (2008). Priming and sentence context support listening to noise-vocoded speech by younger and older adults. J. Acoust. Soc. Am. 123, 489-499. doi: 10.1121/1.2783762

Smiljanic, R., and Sladen, D. (2013). Acoustic and semantic enhancements for children with cochlear implants. J. Speech Lang. Hear. Res. 56, 1085-1096. doi: 10.1044/1092-4388(2012/12-0097)

Stelmachowicz, P. G., Hoover, B. M., Lewis, D. E., Kortekaas, R. W. L., and Pittman, A. L. (2000). The relation between stimulus context, speech audibility, and perception for normal hearing and hearing-impaired children. J. Speech Lang. Hear. Res. 43, 902-914. doi: 10.1044/jslhr.4304.902

Stuart, A. (2005). Development of auditory temporal resolution in schoolage children revealed by word recognition in continuous and interrupted background noise. Ear Hear. 26, 78-88. doi: 10.1097/00003446-20050200000007

Stuart, A., Givens, G. D., Walker, L. J., and Elangovan, S. (2006). Auditory temporal resolution in normal-hearing preschool children revealed by word recognition in continuous and interrupted noise. J. Acoust. Soc. Am. 119, 1946-1949. doi: $10.1121 / 1.2178700$

Wang, J., Shu, H., Zhang, L., Liu, Z., and Zhang, Y. (2013). The roles of fundamental frequency contours and sentence context in Mandarin Chinese speech intelligibility. J. Acoust. Soc. Am. 34, EL91-EL97. doi: 10.1121/1.4811159

Wang, W. S. Y. (1973). The Chinese language. Sci. Am. 228, 50-60. doi: 10.1038/ scientificamerican0273-50

Wermke, K., Ruan, Y., Feng, Y., Dobnig, D., Stephan, S., Wermke, P., et al. (2016). Fundamental frequency variation in crying of Mandarin and German neonates. J. Voice 31, 255.e25-255.e30. doi: 10.1016/j.jvoice.2016.06.009

Wilson, R. H., Farmer, N. M., Gandhi, A., Shelburne, E., and Weaver, J. (2010). Normative data for the words-in-noise test for 6- to 12-year-old children. J. Speech Lang. Hear. Res. 53, 1111-1121. doi: 10.1044/1092-4388(2010/090270)

Xu, G., Zhang, L., Shu, H., Wang, X., and Li, P. (2013). Access to lexical meaning in pitch-flattened Chinese sentences: an fMRI study. Neuropsychologia 51, 550-556. doi: 10.1016/j.neuropsychologia.2012.12.006

Zhang, L., Li, Y., Wu, H., Li, X., Shu, H., Zhang, Y., et al. (2016). Effects of semantic context and fundamental frequency contours on Mandarin speech recognition by second language learners. Front. Psychol. 7:908. doi: 10.3389/fpsyg.2016. 00908

Conflict of Interest Statement: The authors declare that the research was conducted in the absence of any commercial or financial relationships that could be construed as a potential conflict of interest.

Copyright (c) 2017 Zhou, Li, Liang, Guan, Zhang, Shu and Zhang. This is an openaccess article distributed under the terms of the Creative Commons Attribution License (CC BY). The use, distribution or reproduction in other forums is permitted, provided the original author(s) or licensor are credited and that the original publication in this journal is cited, in accordance with accepted academic practice. No use, distribution or reproduction is permitted which does not comply with these terms. 